\title{
Auxin-ethylene transcriptional crosstalk in Arabidopsis thaliana $\mathbf{L}$.
}

Omelyanchuk N.A. ${ }^{1 *}$, Ubogoeva E.V. ${ }^{1}$, Zemlyanskaya E.V. ${ }^{1,2}$

${ }^{1}$ Institute of Cytology and Genetics, SB RAS, Novosibirsk, Russia

${ }^{2}$ Novosibirsk State University, Novosibirsk, Russia

*email:nadya@bionet.nsc.ru

Phytohormones auxin and ethylene are key regulators of plant development and responses to environmental changes targeting these processes independently or together. Many aspects of interplay between auxin and ethylene were described but systematic overview assessing intensity of their interaction (existence of common targets, involvement in regulation of partner metabolism or signaling) is still lacking. Herein, we investigated auxin-ethylene transcriptional crosstalk at whole-genome resolution carrying out meta-analysis of transcriptome changes in Arabidopsis thaliana triggered by external application of auxin or ethylene.

Clustering of auxin and ethylene responsive transcriptomes segregated them according to treatment duration and organ specificity. Gene ontology (GO) annotation of differentially expressed genes (DEGs) in the clusters unveiled involvement of some of them in processes regulated by both hormones similarly or antagonistically. For example, both ethylene and auxin upregulate chitin and cadmium responses. Otherwise, auxin upregulates whereas ethylene downregulates unidimensional cell growth. Besides, both hormones regulate response to each other and cooperate in the control of responses to other hormones, cold and salt stresses, and development (cell wall organization and root hair elongation).

Genes differentially expressed in response to both hormones (common targets) were classified according to direction of expression changes (up- or downregulation). GO annotation of these groups identified processes enriched with common targets regulated in concert by auxin and ethylene. Intersecting the results on functional annotation of DEGs and common targets resulted in subdivision of processes regulated by both hormones into enriched and not enriched with common targets.

We extracted from the list of auxin induced DEGs genes involved in ethylene metabolism and signaling and vice versa. We left in both lists only genes, which involvement has references to the published papers. Then we embed these "hidden" interactions into the processes regulated by auxin and ethylene. Thus, we provide a roadmap of auxin-ethylene crosstalk at the transcriptional level integrating all main aspects of their interactions.

Acknowledgments: This work was supported by the RSF grant 20-14-00140. 